\title{
Research on the Effect of Superficial HFUS Combined with ERUS on the Diagnostic Accuracy of Patients with Perianal Abscess
}

\author{
Zheng Wang \\ Department of Ultrasound, Henan Province Traditional Chinese Medicine Hospital, Zhengzhou, 450002, \\ China
}

Keywords: Superficial HFUS; ERUS; Perianal Abscess; Accuracy

\begin{abstract}
Objective: To analyze the effect of superficial high frequency ultrasound (HFUS) combined with transrectal ultrasound (ERUS) on the diagnostic accuracy of patients with perianal abscess. Methods: Sixty-three patients with perianal abscess were selected from June 2017 to August 2018. 48 cases of simple perianal abscess, 10 cases of anal fistula, and 5 cases of compound perianal abscess were confirmed by operation. Among them, 13 cases of perianal abscess were formed. There were 38 cases of perianal cyst formation and 12 cases of perianal abscess formation. The superficial HFUS, ERUS and superficial HFUS+ERUS tests were performed, and the accuracy of the above test methods was counted. Results: The accuracy of different types of perianal abscess was $92.06 \%$ higher than that of superficial HFUS 66.67\% and ERUS 68.25\% $(\mathrm{P}<0.05)$. The accuracy of combined diagnosis of perianal abscess in different periods was $90.48 \%$ higher than superficial HFUS 69.84\%, ERUS $68.25 \%$ was diagnosed alone $(\mathrm{P}<0.05)$. Conclusion: The superficial HFUS combined with ERUS is applied to patients with perianal abscess, which can effectively improve the diagnostic accuracy and provide a scientific and reasonable reference and basis for clinical selection of surgical timing, planning of surgical procedures and observation of therapeutic effects.
\end{abstract}

\section{Introduction}

Perianal abscess is an acute suppurative infection, which mainly occurs in the soft tissue around the anorectal rectum. It has the characteristics of insidious onset, repeated illness, prolonged course of disease, etc. If the diagnosis and treatment are not timely, it is easy for the patient's daily life and work [1]. Anal digital examination and rectal examination as a common method for clinical diagnosis of perianal abscess, more invasive operation, coupled with severe pain in the perianal area, the patient is unbearable, seriously affecting the diagnosis and treatment effect. With the continuous improvement and optimization of ultrasonic diagnostic technology, it is widely used in the clinical diagnosis of perianal abscess, with significant advantages such as high image resolution, convenient operation and high precision [2]. In this study, 63 patients with perianal abscess were selected to investigate the clinical value of super high frequency ultrasound (HFUS) combined with endoscopic ultrasonography (ERUS). The details are as follows.

\section{Materials and Methods}

\subsection{General Information.}

From June 2017 to August 2018, 63 patients with perianal abscess were enrolled, including 26 females and 37 males, aged 18-64 years, with an average age of (45.15 \pm 7.18$)$ years. Surgery results confirmed 48 cases of simple perianal abscess, 10 cases of anal fistula, 5 cases of compound perianal abscess, including 13 cases of perianal abscess formation, 38 cases of perianal cyst formation, 12 cases of perianal abscess formation. This study meets the relevant requirements of the Helsinki Declaration of the World Medical Association and is approved by the Ethics Committee of our hospital. 


\subsection{Selection Criteria.}

(1) Inclusion criteria: patients and their families informed and signed consent forms; all were accompanied by clinical manifestations such as limited sitting activity and perianal local pain; (2) Exclusion criteria: severe heart, brain, liver and kidney, lung Such organ failure; coagulation system abnormalities; severe organic lesions; associated with malignant tumors; mental problems or inability to communicate and communicate properly; accompanied by cardiovascular and cerebrovascular diseases, those with physical activity disorders; ; with autoimmune diseases.

\subsection{Methods.}

Patients were given hypnosis 24 hours before the test [mannitol (oral) + sodium chloride injection (0.9\%, intravenous drip)], cleansing enema [ metronidazole + sodium chloride injection $(0.9 \%)]$, etc. Female patients were examined in the non-menstrual period, and the left lateral position was performed. The perianal palpation and rectal examination were used to understand the specific conditions (size, location, morphology, and abscess) of the perianal abscess.

The instrument adopts color Doppler ultrasonic instrument DC-3 (supplied by Jiuzhou Tong Pharmaceutical Group Co., Ltd.), with a superficial HFUS probe frequency of 7 to $15 \mathrm{MHz}$ and an ERUS probe frequency of 5 to $11 \mathrm{MHz}$.

Superficial HFUS test probe is applied with couplant, cover the probe with isolation sleeve, center the anus, scan counterclockwise or timely for the perianal (multi-angle, multi-section), fully display the anal canal and surrounding Tissue anatomy, close monitoring of abnormal echo and blood flow signals, positioning and description of lesion location, size, lesion connection, internal echo, fistula (part, number) and shape according to the clock method, and using color Doppler Blood flow imaging (CDFI) carefully explores the blood flow of the lesion and records relevant data.

ERUS inspection probe application couplant, double-layer condom cover the probe, slowly push into the rectum, using the clock method (counterclockwise or clockwise) on the anorectal cavity and surrounding tissue specific conditions [focal position, size, lesion contact, internal echo, fistula (part, number) and shape, etc.] multi-section, circular scan, while CDFI to carefully explore the blood flow of the lesion, and record relevant data.

\subsection{Diagnostic Criteria.}

Exploring the anal canal soft tissue visible echo group (even), irregular shape, blurred boundary, and more blood flow signals were detected by CDFI to explore the lesion and surrounding tissues for the early stage of perianal abscess formation; due to liquefaction necrosis of tissue inside the abscess, Therefore, the observation was based on no echo, the boundary definition was high, and the wall of the capsule was thick but uneven. The blood flow signal was observed by CDFI to detect the wall of the abscess, but the blood flow signal at the necrosis site was the period of perianal abscess formation; the lesion was not found. Uniform hypoechoic, clear boundary, no blood flow signal detected by CDFI for the late stage of perianal abscess formation.

\subsection{Observation Indicators.}

(1) Superficial HFUS, ERUS and combined examination of different types of perianal abscess results. (2) Superficial HFUS, ERUS and combined examination of perianal abscess results in different periods.

\subsection{Statistical Analysis.}

The data were analyzed by SPSS25.0 software. The count data was expressed by n(\%), and the $\chi 2$ test was performed. $\mathrm{P}<0.05$ was considered statistically significant. 


\section{Results}

\subsection{The Results of Different Types of Perianal Abscess.}

Combined with diagnosis of perianal abscess were 92.06\% higher than superficial HFUS 66.67\% and ERUS 68.25\% $(\mathrm{P}<0.05)$, and the combined diagnosis Kappa index was higher than superficial HFUS and ERUS alone. See Tables 1, 2.

Table 1 superficial HFUS, ERUS alone and combined diagnosis of different types of perianal abscess

\begin{tabular}{|c|c|c|c|c|c|c|c|c|c|c|}
\hline \multirow[b]{2}{*}{$\begin{array}{l}\text { Different } \\
\text { kinds }\end{array}$} & \multicolumn{3}{|c|}{ Superficial HFUS } & \multicolumn{2}{|l|}{ ERUS } & \multicolumn{4}{|c|}{ Joint inspection } & \multirow[b]{2}{*}{ Total } \\
\hline & $\begin{array}{l}\text { Simple } \\
\text { type }\end{array}$ & $\begin{array}{l}\text { Anal } \\
\text { fistula }\end{array}$ & Compound & $\begin{array}{l}\text { Simple } \\
\text { type }\end{array}$ & $\begin{array}{l}\text { Anal } \\
\text { fistula }\end{array}$ & $\begin{array}{l}\text { Compo } \\
\text { und }\end{array}$ & $\begin{array}{l}\text { Simple } \\
\text { type }\end{array}$ & $\begin{array}{l}\text { Anal } \\
\text { fistula }\end{array}$ & Compound & \\
\hline $\begin{array}{l}\text { Simple } \\
\text { type }\end{array}$ & 36 & 10 & 2 & 34 & 9 & 5 & 46 & 2 & 0 & 48 \\
\hline $\begin{array}{l}\text { Anal } \\
\text { fistula }\end{array}$ & 4 & 5 & 1 & 2 & 7 & 1 & 1 & 9 & 0 & 10 \\
\hline Compound & 1 & 3 & 1 & 1 & 2 & 2 & 0 & 2 & 3 & 5 \\
\hline Total & 41 & 18 & 4 & 37 & 18 & 8 & 47 & 13 & 3 & 63 \\
\hline
\end{tabular}

Table 2 superficial HFUS, ERUS alone and combined diagnosis of different types of perianal abscess accuracy n (\%)

\begin{tabular}{lllll}
\hline Diagnostic method & Accuracy (\%) & Kappaindex & $95 \%$ confidence interval & $P$ \\
\hline Superficial HFUS & $66.67(42 / 63)$ & 0.283 & $0.087 \sim 0.481$ & 0.000 \\
ERUS & $68.25(43 / 63)$ & 0.361 & $0.182 \sim 0.540$ & 0.000 \\
Joint diagnosis & $92.06(58 / 63)$ & 0.799 & $0.600 \sim 0.998$ & 0.000 \\
$\chi^{2}$ & 13.849 & & & \\
$P$ & 0.001 & & & \\
\hline
\end{tabular}

\subsection{Peripheral Abscess Results of Different Periods of Combined Examination of Perianal} Abscess.

In different periods of accuracy of 90.48\% higher than superficial HFUS 69.84\%, ERUS 68.25\% alone $(\mathrm{P}<0.05)$, and combined diagnosis Kappa index is higher than superficial HFUS, ERUS alone For diagnosis, see Tables 3 and 4.

Table 3 superficial HFUS, ERUS alone and combined diagnosis of perianal abscess results in different periods

\begin{tabular}{|c|c|c|c|c|c|c|c|c|c|c|}
\hline \multirow{2}{*}{$\begin{array}{l}\text { At } \\
\text { times }\end{array}$} & \multicolumn{3}{|c|}{ Superficial HFUS } & \multicolumn{3}{|l|}{ ERUS } & \multicolumn{3}{|c|}{ Joint inspection } & \multirow[b]{2}{*}{ total } \\
\hline & $\begin{array}{l}\text { Pre-forma } \\
\text { tion }\end{array}$ & $\begin{array}{l}\text { Formation } \\
\text { period }\end{array}$ & $\begin{array}{l}\text { Late } \\
\text { formation }\end{array}$ & Pre-formation & $\begin{array}{l}\text { Formation } \\
\text { period }\end{array}$ & $\begin{array}{l}\text { Late } \\
\text { formation }\end{array}$ & Pre-formation & $\begin{array}{l}\text { Formation } \\
\text { period }\end{array}$ & $\begin{array}{l}\text { Late } \\
\text { formation }\end{array}$ & \\
\hline Pre-formation & 9 & 4 & 0 & 8 & 4 & 1 & 11 & 2 & 0 & 13 \\
\hline $\begin{array}{l}\text { Formation } \\
\text { period }\end{array}$ & 9 & 27 & 2 & 8 & 29 & 1 & 2 & 36 & 0 & 38 \\
\hline Late formation & 1 & 3 & 8 & 0 & 6 & 6 & 0 & 2 & 10 & 12 \\
\hline total & 19 & 34 & 10 & 16 & 39 & 8 & 13 & 40 & 10 & 63 \\
\hline
\end{tabular}

Table 4 Superficial HFUS, ERUS alone and combined diagnosis of perianal abscess accuracy in different periods $\mathrm{n}(\%)$

\begin{tabular}{lllll}
\hline Diagnostic method & Accuracy(\%) & Kappa index & $95 \%$ confidence interval & $P$ \\
\hline Superficial HFUS & $69.84(44 / 63)$ & 0.482 & $0.303 \sim 0.661$ & 0.000 \\
ERUS & $68.25(43 / 63)$ & 0.423 & $0.241 \sim 0.605$ & 0.000 \\
Joint diagnosis & $90.48(57 / 63)$ & 0.825 & $0.643 \sim 1.007$ & 0.000 \\
$\chi^{2}$ & 10.675 & & & \\
$P$ & 0.005 & & & \\
\hline
\end{tabular}




\section{Discussion}

The clinical manifestations of perianal abscess are generalized fatigue, chills and other symptoms, often accompanied by systemic poisoning symptoms. If the perianal abscess is not treated in time or improperly treated, it will not only aggravate the patient's pain, increase the economic burden, but also prone to the spread of infected lesions [3]. Surgical treatment is the most direct and effective treatment for clinical treatment of perianal abscess. It can effectively promote pus drainage, completely remove the lesion, and prevent or reduce the spread of infection lesions [4]. Therefore, early diagnosis and treatment of perianal abscess can help to avoid the risk of anal fistula formation and improve the quality of life of patients. At present, the commonly used clinical examination methods are doctor palpation, probes, etc. Although it has the advantages of simple operation and low cost, it has strong subjectivity and low accuracy, and cannot effectively judge the position and size of the lesion [5].

Superficial HFUS is applied to superficial perianal lesions with high resolution, which can clearly show the specific conditions of the lesion (morphology, size, location, number and blood supply relationship with surrounding tissues), and its application to liquid lesions, diagnostic sensitivity and The accuracy rate is higher. In addition, it can accurately stage the perianal abscess by the size of abscess, pus trait and wall thickness of the abscess (pre-formation, formation stage, late stage of formation), thus providing certain science for clinical selection of different treatment options. Refer to [6]. However, studies have shown that superficial HFUS is difficult to effectively display deep abscesses, and it is easy to ignore small abscesses in deep areas [7]. ERUS can effectively compensate for the deficiency of superficial HFUS, and it can be more intuitive and clear exploration of the depth of abscess (high perianal abscess), size and structure of surrounding tissues. At the same time, ERUS can accurately locate the position of anal fistula and show the anal fistula. The number of fistulas, etc., in addition to its high accuracy of internal position, can effectively promote the three-dimensional lesions [8]. Superficial HFUS combined with ERUS combines the advantages of both, and exerts the advantage of positioning and positioning, which can effectively diagnose and treat perianal abscess as early as possible, help to reduce the incidence of postoperative complications and avoid secondary surgery. In this study, superficial HFUS combined with ERUS was applied to patients with perianal abscess. The results showed that the accuracy of different types of combined diagnosis of perianal abscess was $92.06 \%$ higher than that of superficial HFUS $66.67 \%$ and ERUS $68.25 \%$, and the accuracy of perianal abscess was $90.48 . \%$ was higher than superficial HFUS 69.84\% and ERUS $68.25 \%$ was diagnosed alone $(\mathrm{P}<0.05)$. It is suggested that the joint examination can effectively improve the diagnostic accuracy.

In summary, superficial HFUS combined with ERUS examination in patients with perianal abscess can effectively improve the diagnostic accuracy provide a scientific, reasonable reference and basis for clinical selection of surgical timing, development of surgical plans, observation of treatment effects.

\section{References}

[1] Zhai Jinghui, Wang Wei. Advances in the application of negative pressure wound treatment technology in perianal abscess [J]. Chinese Journal of Emergency Medicine, 2016, 25 (1): 106-109.

[2] Zhang Hui, Xiang Sufang, Song Jun, et al. Therapeutic effect of high frequency ultrasound guided minimally invasive treatment of superficial perianal abscess [J].Journal of Practical Hospital, 2015, 12(3):114-116.

[3] Feng Yuening, Feng Dayong, Wang Chunhui, et al. Clinical observation of self-made Qingrejiedufang fumigation and washing for perianal abscess after single radical resection [J]. Chinese Journal of Emergency Medicine, 2016, 25(8): 1603-1605.

[4] Li Jianfeng, Chang Linwei, Zheng Yongliang. Effect of Kangfu Xiaoyan suppository combined with stage I radical operation on perianal abscess [J]. Chinese Medicine Herald, 2017, 23 (15): 98-100. 
[5] Wang Pengzhen, Zeng Yanrong. The value of high-frequency linear array and transrectal ultrasound in judging the internal mouth and classification of perianal abscess [J]. Colorectal and anal surgery, 2018, 24 (1): 71-74.

[6] Mo Zelai, Lu Hailan, Lu Liwei, et al. Ultrasound diagnosis of perianal abscess combined with high-frequency superficial small organ and intracavitary probe[J].Chinese Journal of Endemiology and Prevention,2017,32(7):834- 834.

[7] He Xiaohua, Yang Shouhe, Zhao Yuehu, et al. Diagnostic evaluation of high frequency color Doppler ultrasound in patients with perianal abscess [J]. Chinese Journal of Nosocomiology, 2017, 27(15): 3529-3532.

[8] Zeng Li, Zhang Baorong, Ma Jibin. Diagnostic value of high frequency ultrasound combined with transrectal ultrasound in perianal abscess and anal fistula [J].Journal of Colorectal and Anal Surgery, 2017, 23(4):525-528. 